\title{
Konturen einer Weiterentwicklung der Didaktik für Sozialpädagogik
}

\author{
Markus N. Sauerwein
}

Eingegangen: 12. Februar 2020 / Angenommen: 6. Juli 2020 / Online publiziert: 10. November 2020 (C) Der/die Autor(en) 2020

Zusammenfassung Angesichts des zunehmenden Einflusses von Scholarisierungsprozessen auf sozialpädagogische Arbeitsfelder und einer damit einhergehenden Kompetenzorientierung, diskutiert der Beitrag die hieraus resultierenden Herausforderungen für eine Didaktik der Sozialpädagogik. Es darf diagnostiziert werden, dass trotz eines immensen Bedarfs an Lehrkräften für Sozialpädagogik, die Didaktik der Sozialpädagogik überraschend wenig Aufmerksamkeit erhält, sowohl in wissenschaftlichen Publikationen aber auch in den Fachschulen für Sozialpädagogik, denn Sozialpädagogik wird zumeist fachfremd unterrichtet. Ein Überblick des gegenwärtigen Diskussionsstandes zeigt, dass zwar Unterrichtsprinzipien und Lehrmethoden einer Didaktik der Sozialpädagogik benannt werden, es aber an eine Qualitätsdiskussion fehlt. Auch findet kein Bezug auf dahinterliegende professionstheoretische Verständnisse statt. Der Beitrag greift dies auf und argumentiert, dass Kompetenzorientierung in der Erzieher*innen-Ausbildung zwar durchaus kritisch betrachtet werden kann, letztlich jedoch nicht zu ignorieren ist. Um hierauf angemessen zu reagieren, sollte die Didaktik der Sozialpädagogik schulpädagogische Konzepte zur Kenntnis nehmen, um eine spezifische sozialpädagogische Antwort auf die Kompetenzorientierung zu entwickeln. Diese könnte etwa in der Entwicklung ,sozialpädagogischer Dimensionen guten Unterrichts ' für Fachschulen für Erzieher*innen bestehen. Zudem sollten Kompetenzen nicht über standardisierte Tests erhoben werden, sondern in der Bearbeitung der Antinomien des beruflichen Handelns, die auf unterschiedlichen Niveaustufen erfolgen kann.

Dr. M. N. Sauerwein $(\bowtie)$

DIPF । Leibniz-Institut für Bildungsforschung und Bildungsinformation, Rostocker

Straße 6, 60323 Frankfurt, Deutschland

E-Mail: sauerwein@dipf.de

Fliedner Fachhochschule Düsseldorf, Geschwister-Aufricht-Straße 9, 40489 Düsseldorf, Deutschland 
Schlüsselwörter Didaktik der Sozialpädagogik · Kompetenzorientierung ·

Erzieher*innen-Ausbildung · Kasuistik · Professionsverständnis

\title{
Outlining an development of didactics for socialpedagogy
}

\begin{abstract}
In view of the increasing influence of the scholarly process on social pedagogical fields of work and the associated competence orientation, the article discusses the resulting challenges for a didactics of social pedagogy. It can be diagnosed that despite an immense demand for teachers for social pedagogy, the didactics of social pedagogy receives surprisingly little attention, both in scientific publications and in the schools for social pedagogy, because social pedagogy is mostly taught outside the subject. An overview of the current state of discussion shows that teaching principles and teaching methods of social pedagogy didactics are mentioned. However there is a lack of a discussion of teaching quality. There is also no reference to the underlying understanding of profession and professionalization. The article takes up this point and argues that although competence orientation in the training of pre-school teachers can be viewed critically, it cannot be ignored. In order to react appropriately to this, the didactics of social pedagogy should take note of concepts of teaching quality in order to develop a specific social pedagogical response to competence orientation. This could consist, for example, in the development of 'socio-pedagogical dimensions of teaching quality' for schools for preschool teachers. In addition, competencies should not be assessed by standardized tests, but rather by working on the antinomies of professional action, which can be done at different levels.
\end{abstract}

Keywords Didactics of social pedagogy · Competence orientation · Pre-school teacher training $\cdot$ Casuistry $\cdot$ Profession and professionalization

Seit rund 20 Jahren ist eine Scholarisierung sozialpädagogischer Arbeitsfelder zu beobachten, die auch Kindertagesstätten inkludiert. Erzieher*innen sind seit der Rezeption der Ergebnisse der ersten PISA-Studie 2000 vermehrt aufgefordert, Aufgaben der Bildungsförderung - und Bildung wird hier durchaus schulisch verstanden - zu übernehmen (König 2016; Liebig 2016; Rudolph 2012; Küls 2010). „Somit rücken didaktische Fragen in den Vordergrund, die sowohl die Gestaltung sozialpädagogischer Arbeit innerhalb der Kindertageseinrichtungen betreffen, als auch in der Ausbildung Berücksichtigung finden sollten“ (Karber 2014, S. 17) und sich praktisch etwa in den Bildungsplänen der Kindertagesstätten widerspiegeln (König et al. 2018; Liebig 2016). In diesen Bildungsplänen werden Kompetenzen definiert, die sich an schulischen Bildungsinhalten orientieren wie bspw. naturwissenschaftliche und mathematische Kompetenzen (Kultusminister Konferenz (KMK) 2017, S. 18). Auch in Forschungsarbeiten ist die gegenwärtige Kompetenzorientierung der Frühpädagogik klar erkennbar. Beispielsweise wird in Abhängigkeit von Qualitätsdimensionen, wie kognitive Aktivierung und konstruktive Unterstützung, die aus der Unterrichts- 
forschung stammen ${ }^{1}$, die Vermittlung mathematischer und naturwissenschaftlicher Inhalte analysiert (u. a. Drexl et al. 2019; Pohle et al. 2019).

Zentrale Referenz und Ausgangslage der Kompetenzorientierung sind nach wie vor die Bildungsstandards (Klieme et al. 2003), in denen Kompetenzen verstanden werden als ,[e]rworbene, also nicht von Natur aus gegebene Fähigkeiten, die an und in bestimmten Dimensionen der gesellschaftlichen Wirklichkeit erfahren wurden und zu ihrer Gestaltung geeignet sind, Fähigkeiten zudem, die der lebenslangen Kultivierung, Steigerung und Verfeinerung zugänglich sind, sodass sie sich intern graduieren lassen, z. B. von der grundlegenden zur erweiterten Allgemeinbildung; aber auch Fähigkeiten, die einen Prozess des Selbstlernens eröffnen, weil man auf Fähigkeiten zielt, die nicht allein aufgaben- und prozessgebunden erworben werden, sondern ablösbar von der Ursprungssituation, zukunftsfähig und problemoffen“ (Klieme et al. 2003, S. 65) sind. In den Bildungsstandards werden Kompetenzen benannt, die Schule Schüler*innen vermitteln sollte, damit bestimmte zentrale Bildungsziele erreicht werden und die letztlich auch empirisch gemessen werden können (Klieme et al. 2003). Kritiker der Kompetenzorientierung (u. a. Zierer 2012; Gruschka 2012, 2011, 2006) argumentieren demgegenüber, dass Kompetenzen als die Aufgliederung und Operationalisierung von Bildung zu verstehen seien. „Die Bildung des Subjekts bleibt [...] im spekulativ Verborgenen und es bleibt nur eine Testierung unterschiedlicher Fähigkeiten übrig, die man gegebenenfalls hierarchisch ordnet [...]“ (Gruschka 2011, S. 49.).

Obwohl Kompetenzorientierung auch in sozialpädagogischen Handlungsfeldern zunehmend virulent und als Herausforderung skizziert wird (Liebig 2016; FröhlichGildhoff et al. 2011), fehlt eine klare Positionierung (der Didaktik) der Sozialpädagogik hierzu (Karber 2014, S. 183). Auch für die Ausbildung der Erzieher*innen müssten hieraus didaktische Konsequenzen resultieren (König 2016). Herausgefordert ist die Didaktik der Sozialpädagogik ferner durch ambivalente Tendenzen; einerseits wird eine Akademisierung der frühen Bildung gefordert - sichtbar durch die Einführung entsprechender Studiengänge für frühkindliche Bildung - andererseits liegen Pläne vor, eine neue Ausbildung unterhalb der für Erzieher*innen zu etablieren $^{2}$. Schließlich darf insgesamt ein enormer Fachkräftemangel konstatiert werden, der sich mit der geplanten Einführung des Rechtsanspruchs auf Ganztagsbetreuung im Jahr 2025 weiter verschärfen wird. In diesem Zusammenhang muss aus sozialpädagogischer Perspektive die Frage aufgeworfen werden, wer die zukünftigen Fachkräfte ausbilden wird und wie.

Der Einfluss der Didaktik der Sozialpädagogik und damit auch der Sozialpädagogik insgesamt scheint jedoch äußerst gering zu sein, wird beachtet, dass an den Fachschulen für Sozialpädagogik drei Viertel der Lehrkräfte fachfremd unterrichten (König 2016). Provokant könnte angesichts dieser Tatsache gefragt werden, ob es

\footnotetext{
1 vgl. hierzu auch das CLASS-Framework (Pianta und Hamre 2009a, 2009b; Hamre et al. 2007), das erstaunliche Übereinstimmung mit den Basisdimensionen guten Unterrichts (Klieme 2019) aufweist.

2 Vgl. hierzu auch die Stellungnahme der Sektion ,Sozialpädagogik und Pädagogik der frühen Kindheit* in der Deutschen Gesellschaft für Erziehungswissenschaft (DGfE) und des Studiengangstages Pädagogik der Kindheit zu der von der KMK geplanten Ausbildung „staatlich geprüfte*r Fachassistent*in für frühe Bildung und Erziehung“.
} 
überhaupt eines eigenständigen Lehramtsstudiums für Fachschulen der Sozialpädagogik bedarf. Fast sarkastisch muss hierauf geantwortet werden, dass vorliegende Forschungsarbeiten diese Frage nicht beantworten und damit (auf empirischer Basis) nicht entkräften können. Es liegen keine Befunde vor, die beispielsweise betrachten, welchen Einfluss die Qualifikation der Lehrkräfte für die Unterrichtsgestaltung und Ausbildung der zukünftigen Erzieher*innen hat (vgl. König 2016).

Der vorliegende Beitrag diskutiert die sich aus dieser Entwicklung abzeichnenden Herausforderungen und Chancen für die Ausbildung der Lehrkräfte der beruflichen Fachschulen für Sozialpädagogik mit dem Ziel, eine konzeptionelle Weiterentwicklung der Didaktik der Sozialpädagogik zu grundieren. Bevor der Forschungs- und Diskussionsstand der Didaktik der Sozialpädagogik rezipiert wird (2), erfolgt eine kurze Ausführung zwei professionstheoretischer Perspektiven (1), die für die nachfolgende Argumentation relevant sind. Anschließend werden Ansätze der Schulund Unterrichtsforschung skizziert, die für eine Weiterentwicklung einer Didaktik der Sozialpädagogik fruchtbar erscheinen (3). Schließlich werden im vierten Abschnitt die zuvor dargelegten Diskussionsstränge zusammengeführt und Konturen einer Weiterentwicklung der Didaktik der Sozialpädagogik dargelegt.

\section{Professionstheoretischer Diskurs}

Verbunden mit diesen eben skizzierten Herausforderungen ist auch das zugrunde liegende Professionsverständnis. Während ein strukturtheoretisches Professionsverständnis die stellvertretende Lösung von Krisen und die Bearbeitung berufsimmanenter Widersprüche betont (Helsper 2014; Wernet 2014; Oevermann 1996), fokussiert ein kompetenzorientiertes Professionsverständnis die Fragen nach den professionellen Kompetenzen, die für die Qualität (von Unterricht) und das Lernen der Heranwachsenden bedeutsam sind (Baumert und Kunter 2006). Dieses letzte Modell geht davon aus, dass professionelles Handeln auf grundlegenden Fähigkeiten basiert, die auf Wissensbestände verweisen, auf die pädagogisch Tätige zurückgreifen (Helsper und Tippelt 2011). Als generisches pädagogisches Wissen und Können wird konzeptuelles bildungswissenschaftliches Grundlagenwissen, allgemeindidaktisches Konzeptions- und Planungswissen, Unterrichtsführung und Orchestrierung von Lerngelegenheiten sowie fachübergreifende Prinzipien des Diagnostizierens, Prüfens und Bewertens aufgeführt (Baumert und Kunter 2006, S. 485). Die strukturtheoretischen Ansätze (in Anlehnung an Oevermann) hingegen gehen von einer Nicht-Standardisierbarkeit professionellen Handelns aus, in dem es darum geht, nicht zu vermeidende Antinomien zu bearbeiten. Pädagogisches Handeln ist demnach widersprüchlich, weil es sich erstens um eine ,stellvertretende, verantwortliche Lebenspraxis für eine andere Lebenspraxis“ (Helsper 2016, S. 53.) handelt, die noch nicht über hinreichend lebenspraktische Autonomie verfügt und zweitens zwei entgegengesetzten Logiken entsprechen muss: der Gleichbehandlung, Begrenzung und Distanzwahrung und zugleich der stellvertretenden Krisenlösung im biografischen Zusammenhang (Helsper 2016). Dies wird etwa deutlich in der Vorgabe, Schüler*innen anhand gleicher Kriterien zu bewerten, sie aber individuell zu fördern, ihre jeweiligen unterschiedlichen Hintergründe zu berücksichtigen, etc. (Helsper 
2007). Werner Helsper (2007) beschreibt insgesamt u. a. folgende Antinomien für pädagogische Berufe:

- Die Begründungsantinomie, als Widerspruch situativ (schnell) handeln zu müssen und zugleich das eigene Handeln zu begründen, für das die eigene Wissensbasis oftmals nicht genügt.

- Die Praxisantinomie, die das hoch belastete und verantwortliche eigene Handeln auf der einen Seite und zugleich eine vom Handlungsdruck befreite wissenschaftliche Reflexion des eigenen Handelns erfordert.

- Die Subsumtionsantinomie, meint die Spannung von verstehendem Nachvollzug für den Einzelfall und der Unterordnung des Einzelfalls unter verallgemeinernde Kategorien.

- Die Ungewissheitsantinomie, als Spagat zwischen dem Erfolgsversprechen des eigenen Handelns und der strukturellen Ungewissheit des eigenen Handelns, da Pädagogen*innen auf die Mitwirkung ihrer Adressat*innen, Schüler*innen etc. für ein erfolgreiches Handeln angewiesen sind.

- Die Symmetrieantinomie, die die ungleiche Positionierung der Professionellen und ihrer Adressat*innen umfasst, die den Zielen des pädagogischen Handelns nach Gerechtigkeit, psychischer Integrität und Bildung entgegensteht.

- Die Vertrauensantinomie, die das widersprüchliche Verhältnis zwischen der Herstellung von Vertrauen als Basis pädagogischer Arbeit auf der einen Seite, aber einer einseitigen Abhängigkeit auf der anderen Seite, umfasst.

Dieser Diskurs scheint in der Didaktik der Sozialpädagogik nicht rezipiert zu werden, obwohl er auch im Zusammenhang mit einer Kompetenzorientierung zu lesen ist und das kompetenzorientierte das strukturtheoretische Professionsverständnis zu verdrängen scheint (Helsper 2007). Sichtbar wird dies beispielsweise, wenn Kompetenzen für zukünftige Erzieher*innen (oder Lehrkräfte) definiert werden und nicht von zu thematisierenden pädagogischen Antinomien gesprochen wird.

\section{Didaktik der Sozialpädagogik}

Die Ausbildung von Lehrkräften für die berufliche Fachrichtung Sozialpädagogik ist derzeit in Deutschland an den Standorten Bamberg, Dresden, Dortmund, Lüneburg, Tübingen und neustens Osnabrück möglich - neu hinzukommen wird voraussichtlich auch Magdeburg. Diese geringe Zahl an Studienorten und -plätzen, scheint dem Bedarf an Lehrkräften für die berufliche Fachrichtung Sozialpädagogik bei weitem nicht gerecht zu werden (Liebig und Gängler 2018; Karber 2014). Dieses Defizit überrascht, ist die Erzieher*innen-Ausbildung, neben dem Studium, die zentrale Qualifikationsmöglichkeit für das Berufsfeld der Sozialen Arbeit respektive Kinder- und Jugendhilfe (Kruse 2017; Beher et al. 1999, S. 5). Ebenso liegen insbesondere im Vergleich zu der sonst regen und vielfältige Publikationslandschaft der Sozialen Arbeit - kaum Veröffentlichungen oder konzeptionelle Entwürfe bzgl. 
der Ausbildung von Lehrkräften oder gar einer Didaktik für Sozialpädagogik vor ${ }^{3}$, sodass Anke Karber (2014) das bestehende Forschungsdesiderat als kennzeichnend für die Didaktik der Sozialpädagogik beschreibt (auch König et al. 2018, S. 32). Es bestehen lediglich einige überblicksartige Arbeiten (Göddertz und Karber 2019; Karber 2014, S. 199; Karsten und Kubandt 2017), wobei wechselseitige Referenzen oder eine Auseinandersetzung von Fachdidaktik und Fachwissenschaft (Cloos und Hoffmann 2001) nicht vorliegen, obwohl dies nach wie vor gefordert, bzw. nach Möglichkeiten einer Verknüpfung gesucht wird (Göddertz und Karber 2019).

Als Begründung wird das eher schwierige, bisweilen konfliktäre, Verhältnis zwischen Sozialpädagogik und Schule aufgeführt (Karber 2017, S. 87; Gängler und Wustmann 2004). Der Begriff Didaktik wird gemeinhin mit der Schulpädagogik in Verbindung gebracht (Steinbacher 2018), scheint vor- und nachschulische Kontexte auszublenden (Terhart 2018) und der Sozialpädagogik wird insgesamt das Vorhandensein einer eigenen Didaktik abgesprochen. Stattdessen habe sich eine ,eigene Methodik entwickelt, die sich aus der Eigenart ihrer Aufgaben und Problembehandlungen ergebe“ (Prange 2011, S. 183). Elke Steinbacher (2018) betont jedoch, dass es in einem weiteren Verständnis von Didaktik um das Lehren und Lernen auch an außerschulischen Orten geht. Bereits Paul Natorp (1899) sprach von Didaktik und betonte in diesem Kontext die Selbsttätigkeit des Lernens: „Hier ist nicht die Rede von einem Einpflanzen von aussen und andererseits passiver Entgegennahme. Die intensivste Förderung durch den Anderen bedeutet vielmehr zugleich intensivste Selbstthätigkeit und umgekehrt“ (Natorp 1899, S. 73).

\subsection{Unterrichtsprinzipien}

Insgesamt mahnt A. Karber (2014) jedoch ,den Mangel an didaktischen Entwürfen“ (Karber 2014, S. 112) an. Dabei sind laut KMK (2017) für die Vermittlung der Kompetenzen und Qualifikationen der zukünftigen Erzieher*innen „,beruflich didaktische Ansätze, die sich von denen anderer beruflicher Ausbildungen unterscheiden“ (KMK 2017, S. 6) nötig, da der Berufsalltag des pädagogischen Arbeitsfelds auf den Umgang mit einzelnen Menschen und Gruppen bezogen ist. Aufgeführt werden diesbezüglich von der KMK (2017) drei Unterrichtsprinzipien ${ }^{4}$ für die beruflichen Schulen für Sozialpädagogik:

a) Der Bezug zum Berufsbereich erfordert eine integrale Persönlichkeitsentwicklung, um den Erziehungs- und Bildungsprozess später in der beruflichen Praxis sinnvoll gestalten zu können. Deshalb ist es wichtig, die Fachschulen bewusst als Lebensund Erfahrungsraum zu gestalten, der die Persönlichkeitsentwicklung fördert.

\footnotetext{
3 Auch in der Qualitätsoffensive Lehrerbildung ist meiner Kenntnis nach - und auch basierend auf dem Überblick von Dietmar Frommberger und Silke Lange (2018) - nur ein Projekt, welches das Lehramt Sozialpädagogik an beruflichen Schulen betrifft (das WegE Projekt in Bamberg; hier liegen jedoch keine Publikationen in Form von zugänglichen Veröffentlichungen vor - nur unveröffentlichte Masterarbeiten).

4 Aus Perspektive der Lehr-Lernforschung darf jedoch allgemein kritisch angemerkt werden, dass Unterrichtsprinzipien normativ aufgeladen sind, wobei Unterrichtsprinzipien auch als Tiefenstruktur des Unterrichts verstanden werden können und entsprechend hier Überschneidungen zur Lehr-Lern-Forschung vorliegen (Reusser 2008).
} 
b) Die Ausbildung muss eine enge Theorie-Praxisverknüpfung sicherstellen. Ausgangspunkt ist die Bearbeitung von sozialpädagogischen Praxissituationen.

c) Unterrichtsprozesse müssen im Sinne der doppelten Vermittlungspraxis so gestaltet sein, dass die angewandten Lehr-/Lernformen auch in der Berufspraxis der späteren sozialpädagogischen Fachkräfte eingesetzt werden können (Karsten und Kubandt 2017; KMK 2017, S. 6f.).

Die von der KMK formulierten Unterrichtsprinzipien betrachten Nina Göddertz und Anke Karber (2019) jedoch in ihrer Auslegung als unzureichend und fordern eine stärkere Berücksichtigung von Gesellschaft und Politik. So sollte das Prinzip der integralen Persönlichkeitsentwicklung Analyse und Kritikfähigkeit beinhalten. Die Theorie Praxis Verknüpfung sollte sich im Modus der Reflexion vollziehen. Lernen bedeutet folglich nicht das Erwerben von Rezeptwissen oder Handlungsanweisungen, sondern bedarf aktiver Neubearbeitung der jeweiligen Situationen. Beides und damit holen N. Göddertz und A. Karber (2019) den doppelten Vermittlungsanspruch ein - sollten die Studierenden als zukünftige Lehrkräfte an beruflichen Schulen nicht nur einüben, sondern auch später in ihrer Lehrtätigkeit selbst anwenden können. Ähnlich betonen auch Hans Gängler und Manuela Liebig (2017) die realen Erfahrungen im Lernprozess, wobei dies mit der Ausrichtung an Lernfelder konfligieren kann (Karber 2014, S. 106), denn wenn nur durch die „Sache selbst“ gelernt wird, braucht es keinen Lehrplan (Gängler und Liebig 2017) oder Lernfelder bzw. es stellt sich die Frage, wie nicht direkt Erfahrenes gelehrt werden kann. Für die Ausbildung der Lehrkräfte für sozialpädagogische Fachschulen besteht in diesem Sinne eine dreifache Vermittlungspraxis: Lehrformen in der Ausbildung der Lehrkräfte für Sozialpädagogik sollten diese selbst im Unterricht einsetzen und diese sollten wiederum von ihren Schüler*innen in sozialpädagogischen Arbeitsfeldern angewendet werden können.

\subsection{Lehrformen und der doppelte Vermittlungsanspruch}

Neben den Unterrichtsprinzipien werden vor allem zwei Lernformen im Kontext der Didaktik für Sozialpädagogik diskutiert - das Forschende Lernen und die Kasuistik, die interessanterweise nur bedingt an ein kompetenzorientiertes Professionsverständnis anschlussfähig ist - auf die nachfolgend eingegangen wird.

\subsubsection{Forschendes Lernen}

Eine Möglichkeit, um den doppelten Vermittlungsanspruch und den Widersprüchen hinsichtlich Kompetenzorientierung und Persönlichkeitsentwicklung begegnen zu können, ist das sogenannte Forschende Lernen (Vriesen 2019; Weyland 2019; Wildt 2005), weil hier Erfahrungen an Lernfelder geknüpft werden könnten. Somit kann auch die Dichotomie Wissenschaftlichkeit und Berufsorientierung aufgelöst (Liebig und Gängler 2018; Karsten 2017) und ein Professionalisierungsprozess angeregt werden (Vriesen 2019). Zugleich können Studierende im wissenschaftlichen Prozess der Erkenntnisgewinnung partizipieren und hierüber auch aktive Selbstaneignungsprozesse ermöglicht werden (Karber und Wustmann 2015). Lerntheoretisch sind hier 
auch Bezüge zu sogenannten konstruktivistischen Lerntheorien erkennbar, deren Fokus auf dem Verstehen und nicht auf dem Behalten von Inhalten liegt (hierzu auch Kunter und Trautwein 2013; Hasselhorn und Gold 2012). Ebenso kann über Forschendes Lernen eine fragend kritisch-reflexive Haltung entwickelt werden (Karber und Wustmann 2015), was auch die von N. Göddertz und A. Karber (2019) geforderte Entwicklung einer Analyse- und Kritikfähigkeit positiv beeinflussen kann.

Kritisch anzumerken ist hierbei jedoch, dass Studierende nicht in jedem Lernfeld forschend tätig sein können, also eher eine Spezialisierung erfolgt. Ebenso kann mit Ulrich Beck und Wolfgang Bonß (1989) argumentiert werden, dass Wissenschaft und Praxis nach unterschiedlichen Rationalitätskriterien handeln und entsprechend jeweils anderes Wissen generiert wird. Dies wird von W. Helsper (2016), wie bereits ausgeführt, auch als Praxisantinomie bezeichnet.

\subsubsection{Kasuistik-Aus Geschichten Lernen}

Eine weitere Lehrform ist das von Andreas Gruschka et al. (1995) beschriebene „Aus Geschichten lernen“ sowie Konzepte der Kasuistik, die auch stärker vordefinierte Lernfelder berücksichtigen können. In diesen Konzeptionen geht es darum, pädagogische Situationen sowie das Handeln der Akteur*innen in diesen Situationen $\mathrm{zu}$ verstehen und für die Lehrer*innen-Bildung zu nutzen (Idel et al. 2014). Gerade die Arbeit an den eigenen Fällen kann hier hilfreich sein, insbesondere für die Lehrkonzeption (Messmer 2014), wobei auf die schulpraktischen Ausbildungskontexte zurückgegriffen werden kann (Idel et al. 2014). Die von A. Gruschka et al. (1995) vorgeschlagene Vorgehensweise erinnert hierbei durchaus an ein - wenn auch stark vereinfachtes - Vorgehen der objektiv hermeneutischen Rekonstruktion, die für den Ausbildungskontext adaptiert wurde und den zukünftigen Erzieher*innen dabei hilft, ihre beruflichen Entwicklungsaufgaben zu bearbeiten (Gruschka 1985; Gruschka et al. 1995). Dabei wird das Handeln in der Praxis als Ausgangspunkt für den Unterricht genommen und Schüler*innen werden aufgefordert, sich reflexiv zu den (eigenen) Praxisbeispielen zu verhalten und diese ,zum Ausgangspunkt einer exemplarischen methodisch streng geregelten Analyse zu machen“ (Gruschka et al. 1995, S. 41). Dieses Vorgehen eignet sich nicht nur für die Ausbildung, sondern kann auch helfen, mit der Praxis produktiv umzugehen und nicht ins Anekdoten erzählen zu verfallen (Gruschka et al. 1995, S. 65). Gleichermaßen für die Ausbildung von Lehrkräften für Sozialpädagogik als auch in der Erzieher*innen Ausbildung kann dies sinnvoll implementiert und damit der doppelte (bzw. dreifache) Vermittlungsanspruch eingeholt werden.

Es geht also darum, sich ,in den Widersprüchen des sozialpädagogischen Dispositivs zu orientieren und kontroverse Sachverhalte diskutieren zu können“ (Hörster 2018, S. 1564). Verknüpft hiermit ist letztlich auch ein strukturtheoretisches Professionsverständnis, das darauf abzielt, die Antinomien des beruflichen Handelns darzulegen (u.a. Helsper 2016, 2007). 


\subsection{Entwicklungsaufgaben und Kompetenzen}

Weder Unterrichtsprinzipien noch Lernformen betrachten den Prozess, wie aus Schüler*innen Erzieher*innen werden. A. Gruschka (1985) hat hierfür das Konzept der beruflichen Entwicklungsaufgaben für Erzieher*innen vorgeschlagen. Er geht davon aus, dass Schüler*innen in Ausbildung und Unterricht nach Hilfestellungen suchen und idealerweise durch diese ihre kognitiven, affektiven und vor allem sozialen Kompetenzen erweitern (Gruschka 1985, S. 46). A. Gruschka (1985) hat insgesamt vier Entwicklungsaufgaben formuliert, die Einschnitte in der Ausbildung zum*zur Erzieher*in markieren und dabei zu ,einer kontinuierlichen Entwicklung seiner sozialberuflichen Kompetenz" (Gruschka 1985, S. 49) beitragen können:

- Konzept einer zukünftigen Berufsrolle, worunter letztlich die Entwicklung eines eigenen Berufsverständnisses mit Erwartungen an die schulische Ausbildung geknüpft ist.

- Das Konzept der pädagogischen Fremdwahrnehmung bewältigen Schüler*innen, wenn es ihnen gelingt, den Rollenwechsel von zu Erziehenden zu Erziehenden zu vollziehen und einen identitätssichernden Zugang zu den Kindern zu finden.

- Das Konzept pädagogisch-praktischen Handelns stellt die Schüler*innen vor die Herausforderung, das eigene Berufsrollenverständnis und die eigene Sicht auf die Adressaten*innen zu einem pädagogischen Handlungsmodell zu integrieren.

- Bei der Strategie der Professionalisierung im Berufsleben sollen Schüler*innen in der Lage sein und die Sicherheit haben, für Probleme des Berufslebens Lösungswege zu finden, ihre eigenen Kompetenzen weiterentwickeln und dabei ihre eigenen Vorstellungen pädagogischer Arbeit umsetzen zu können.

Kompetenz wird hier verstanden als „,die Fähigkeit des Subjektes, sich zur Lösung der Aufgaben zu antizipieren und erfahrene Qualifikationen auf Basis der eigenen Erwartungen anzuzeigen" (Gruschka 1985, S. 53). Es beinhaltet also die Fähigkeit der Schüler*innen zu entscheiden, in welcher Situation sie welche Qualifikation einsetzen. A. Gruschka (1985) formuliert hier bereits - ganz im Sinne seiner späteren kritischen Positionierung gegenüber der empirischen Bildungsforschung - dass bei der Messung von Kompetenzen ein gravierendes methodologisches Problem besteht, da „bestimmte in einer Skala objektivierte Standards einer Problemlösung nicht unbedingt als Ziele der eigenen Entwicklung im Erwartungshorizont der Schüler verankert sind“" (Gruschka 1985, S. 55). Als Lösung - und dies überrascht, wenn man sich die späteren Veröffentlichungen von A. Gruschka vor Augen hält - werden in der Arbeit unterschiedliche Elaborationsstufen der Kompetenzen aus den Aufgaben der Schüler*innen für jede der vier Entwicklungsaufgaben objektiv hermeneutisch rekonstruiert. Interessant ist hierbei, dass A. Gruschka zeigen kann, dass Schüler*innen oft über das entsprechende Wissen verfügen, die Entwicklungsaufgaben zu bewältigen, ihnen aber der Zugang zu den Problemen und Widersprüchen beruflichen Handelns fehlt. In diesem Sinne scheinen Kompetenzen und Fähigkeiten alleine nicht zu genügen und die Entwicklungsaufgaben sollten um die Bearbeitung pädagogischer Antinomien ergänzt werden (siehe Abschn. 4). 


\section{Ansätze der Schul- und Unterrichtsforschung als Anregung einer Weiterentwicklung einer Didaktik der Sozialpädagogik}

Obzwar auf konzeptioneller Ebene dargestellt werden kann, wie die Aspekte des Forschenden Lernens und der Kasuistik mit den Unterrichtsprinzipien harmonieren, ist weitestgehend unklar, wie mit den widersprüchlichen Forderungen nach Kompetenzorientierung, Persönlichkeitsentwicklung, aber auch den zugrundeliegenden professionstheoretischen Verständnis umzugehen ist sowie der Vorbereitung auf die Antinomien im späteren pädagogischen Handeln. Auch A. Gruschkas (1985) Arbeit kann dahingehend gelesen werden, dass die Entwicklung eines professionellen Selbstverständnisses eben nicht durch Praktika und Wissensinhalte vollständig gelingen kann. Ebenso ist unklar, inwiefern die nun über 35 Jahre alten Befunde noch Gültigkeit beanspruchen können.

Letztlich besteht so betrachtet ein Forschungsdesiderat hinsichtlich:

a. des Zusammenhangs der Unterrichtsprinzipien, der Lehrmethoden und der Qualität, in der diese dargeboten werden

b. des Einflusses dieser auf die Bewältigung der beruflichen Entwicklungsaufgaben bzw. dem Erreichen von Kompetenzen (wie bspw. im kompetenzorientierten Qualifikationsprofil aufgeführt; u. a. professionelle Haltung, Sozialkompetenz und Selbständigkeit) sowie der Vorbereitung auf die Bearbeitung der berufsimmanenten pädagogischen Antinomien (sowohl für die zukünftigen Lehrkräfte an Fachschulen für Sozialpädagogik als auch für die Erzieher*innen).

Dieses Forschungsdesiderat kann der vorliegende Beitrag nicht mit eigenen empirischen Erkenntnissen füllen, jedoch wird der Versuch unternommen, eine konzeptionelle Neuorientierung darzulegen, auf die zukünftige empirische Arbeiten rekurrieren können. Hierfür wird zunächst ein „Blick über den Tellerrand“ gewagt und sich bei der Schulpädagogik umgeschaut. Zunächst wird der Ansatz des Verstehen Lehrens von Gruschka thematisiert (Abschn. 3.1). Anschließend wird auf die sogenannte (quantitative) Lehr-Lernforschung eingegangen und das Verhältnis zwischen Unterrichtsqualität und Lehrmethoden dargestellt (Abschn. 3.2), bevor abschließend auf Gemeinsamkeiten und Unterschiede der Konzepte eingegangen wird (Abschn. 3.3) ${ }^{5}$. Aufbauend hierauf werden Konturen einer Weiterentwicklung der Didaktik der Sozialpädagogik erläutert (Abschn. 4).

\subsection{Verstehen Lehren}

A. Gruschkas (2013, 2017) Ansatz kann als eine Weiterentwicklung der allgemeinen Didaktik im Sinne von Lernen als Verstehensprozess beschrieben (Prange 2011) und als Kritik an der Empirischen Bildungsforschung gelesen werden. Unterricht besteht

\footnotetext{
5 Ich beschränke mich hier auf diese Ansätze, da ihr Ziel letztlich gelingender Unterricht bzw. Bildung oder Kompetenzentwicklung darstellt. Natürlich gibt es auch (soziologische) Ansätze, die Wesensmerkmale des Unterrichts beschreiben bzw. ihn hinsichtlich ihrer gesellschaftlichen Funktion analysieren. Dies scheint im Kontext der vorliegenden Arbeit nicht zielführend (zur Übersicht: Breidenstein 2010).
} 
für ihn aus den Dimensionen Erziehung (a), Didaktik (b) und Bildung (c), wobei diese unterschiedlich ausgeprägt, bzw. ,skaliert“ (Klieme 2019) sein können:

a) Erziehung bezieht sich zunächst auf die verhaltensbezogenen Voraussetzungen für erfolgreiches Lernen (Gruschka 2013), die vom notwendigen Umgang abweichenden Verhaltens bis zur Aufhebung der Erziehung durch Erziehung reicht. Diesen Aspekt hatte bereits P. Natorp (1899) betont, indem Erziehung nicht (nur) als Belehrung, sondern als „freie Einsicht“ (Natorp 1899, S. 77) zu verstehen sei.

b) Didaktische Operation versteht A. Gruschka (2013) als den Prozess, die Inhalte des Curriculums an die Schüler*innen weiterzugeben. „Indem die Didaktik einen Repräsentanten der Welt konstruiert, steht sie vor der Aufgabe, das zu Vermittelnde in der Vermittlung aufzuheben, es also zu transformieren und doch als das, was mit der Vermittlung vermittelt werden soll, erkennbar zu machen und zu belassen“ (Gruschka 2013, S. 183). Auch hier beschreibt A. Gruschka (2013) fünf Stufen von Didaktik. Die niedrigsten Stufen sind Entsorgung (der Gegenstand ist nicht mehr als solcher fassbar) und Verfälschung (die Vereinfachung schlägt fehl). In den höheren Stufen geht es um Mitdenken und Reflexion.

c) Didaktik und Erziehung sind auf die Ermöglichung von Bildung ausgerichtet (Gruschka 2013, S. 206). Ebenfalls werden wieder verschiedene Stufen beschrieben, wobei die Faszination und Arbeit an der Erkenntnis als die zwei höchsten Stufen beschrieben werden. Dies liegt vor, wenn Neugier und Erkenntnisinteresse der Schüler*innen geweckt werden und sich Krisen des Verstehens mit Einsicht und Erkenntnis abwechseln.

Empirisch findet A. Gruschka $(2013,2017)$ in seinen Unterrichtsbeobachtungen jedoch kaum die höchsten Stufen von Bildung. Entsprechende Versuche werden zumeist kurz vorher von Lehrkräften abgebrochen, da sie das Schema des vorgegebenen Lehrplans zu verlassen drohen.

\subsection{Lehr-Lern-Forschung/Unterrichtsqualität}

Bildung ist nicht nur in der allgemeinen Didaktik Kernziel, sondern auch aus Perspektive von (einigen) Vertretern*innen der Lehr-Lern-Forschung sollte Unterricht Bildung ,als Aneignung von Kultur und Entfaltung einer mündigen Persönlichkeit““ (Klieme 2019) ermöglichen.

Trotz dieser ähnlichen Zielvorstellung, geht die Lehr-Lernforschung anders vor, da die Wirkungszusammenhänge zwischen Lehren, Lern- und Erfahrungsprozessen der Schüler*innen im Fokus stehen (Terhart 2018) und somit der Bildungsbegriff höchstens theoretisch, aber empirisch durch den handhabbaren Kompetenzbegriff ersetzt wird. Qualität des Unterrichts ist dann vorhanden, wenn die Ziele des Unterrichts erreicht werden (Sauerwein und Klieme 2016). Fragen nach der Struktur und Rechtfertigung von Bildungsinhalten sind empirisch nur begrenzt zugänglich und werden eher randständig thematisiert (Reusser 2008). Eckhard Klieme (2019) kann in seinen Forschungsarbeiten (u. a. Praetorius et al. 2018; Klieme und Rakoczy 2008; Klieme et al. 2001) drei generische Dimensionen von Unterrichtsqualität finden und beschreiben - Classroom Management (a), Motivationsunterstützung (auch schüler*innenorientiertes Unterrichtsklima genannt) (b) und kognitive Aktivierung (c): 
a) Verkürzt dargestellt meint Classroom Mangement die möglichst störungsarme und reibungslose Unterrichtsführung. Es geht demnach auch darum, die vorhandene Unterrichtszeit für das Unterrichten zu nutzen.

b) Motivationsunterstützung beschreibt das Ausmaß, in dem Lehrer*innen Schüler*innen im Lernprozess begleiten und inkludiert die Schüler*innen-Lehrer*innen-Beziehung. Letztlich ist Motivationsunterstützung auf die Ermöglichung eines eigenständigen und selbstbestimmten Lernens und einer entsprechenden hierfür notwendigen Lernumgebung ausgerichtet.

c) Kognitive Aktivierung hingegen beschreibt einen Unterricht, der zum aktiven Nachdenken anregt und letztlich tiefergehende Lernprozesse ermöglichen soll.

In den Forschungsarbeiten von E. Klieme und Kolleg*innen zeigen sich Unterschiede in der Kompetenzentwicklung von Schüler*innen, die auf diese drei Dimensionen zurückgeführt werden können. Zudem scheinen die Qualitätsdimensionen aufeinander aufzubauen. Ein Mindestmaß an Classroom Mangement ist demnach notwendig, um überhaupt kognitiv aktivierend unterrichten zu können (Pietsch 2010). Diese drei Dimensionen finden sich in ähnlicher Variante auch im Dynamic Model of Educational Effectiveness (Kyriakides et al. 2013; Creemers und Kyriakidēs 2008) und im CLASS-Framework (Pianta und Hamre 2009a) wieder, welches auch im Bereich der Kindertagesstätten in adaptierter Form Anwendung findet (Sandilos et al. 2014; Pianta et al. 2008).

\subsection{Gemeinsamkeiten der schulpädagogischen Ansätze}

Auch wenn die Arbeiten von A. Gruschka und E. Klieme methodisch (und methodologisch) von verschieden Perspektiven aus Unterricht betrachten, können einige Gemeinsamkeiten beschrieben werden (Klieme 2019). Jeweils ist ein Mindestmaß an Erziehung bzw. Klassenführung (Classroom management) notwendig, damit überhaupt Unterricht stattfinden kann. Auch lassen sich in A. Gruschkas Unterrichtsbeschreibungen wiederkehrend Phasen dessen finden, was E. Klieme als kognitive Aktivierung bezeichnet: „Die Schüler wechseln selbstständig die Perspektive und beginnen damit, nach Bedeutungen der Textteile zu suchen. Zugleich starten sie immer wieder neue Versuche, durch das Vortragen und Hören das Rätsel des Textes zu lösen“ (Gruschka 2011, S. 163). Während A. Gruschka - ähnlich wie bereits in seiner Erzieher*innen Studie - Niveaustufen des Unterrichts beschreibt, ,ratet“ oder schätzt die Lehr-Lern-Forschung Unterrichtsqualität ein. Die jeweiligen Dimensionen, bei A. Gruschka Didaktik und Bildung genannt, in der Lehr-Lern-Forschung Motivationsunterstützung und kognitive Aktivierung, scheinen hierbei jedoch Ähnliches, wenn nicht sogar das Gleiche, zu erfassen (Sauerwein 2017). 


\section{Konturen einer Weiterentwicklung einer Didaktik für Sozialpädagogik}

Diese schulpädagogischen Grundlagen werden nachfolgend zusammen mit den bereits aufgeführten Ansätzen einer Didaktik der Sozialpädagogik herangezogen, um eine Weiterentwicklung dieser zu skizzieren.

\subsection{Verstehen rekonstruieren}

Rekonstruktionen von pädagogischen Situationen können herangezogen werden, um Verstehensprozesse im Unterricht als Telos von Bildung darzustellen (Gruschka 2017). Verstehen bzw. Irritation für nicht Verstandenes ist hier zentrales Element von Unterricht und Bildung (Gruschka 2017). Das kindliche „Warum?“ müsste bei den zukünftigen Lehrkräften selbst wiedererweckt werden, um im Kontext der doppelten Vermittlungspraxis, Verstehen zu lehren bzw. Nicht-Verstehen als Ausgangspunkt des Verstehens zu begreifen - wie es eben bei Kindern (noch) der Fall ist und dies an die zukünftigen Erzieher*innen weitergeben werden. Verstehen ist die Einheit von Vermittlung und Aneignung. ,Kinder haben, bis sie in die Schule kommen, massenhaft solche Erlebnisse gehabt“, in der Schule ist dies jedoch ein ,eher unwahrscheinliches Ereignis“ (Gruschka 2017, S. 89). Diese Paradoxien sind gerade vor dem Hintergrund einer Scholarisierung außerschulischer Lebenswelten zu bearbeiten. Kontrastiv können sowohl in der Ausbildung für Lehrkräfte der Fachrichtung Sozialpädagogik, aber auch für zukünftige Erzieher*innen Verstehensprozesse in Schulen und Kindertagesstätten rekonstruiert werden. In Anlehnung an P. Natorp (1899) könnte sich in solchen Rekonstruktionen den Verhältnissen zwischen Erziehung, Bildung und Gemeinschaft, die in dieser Konstellation erst hervortreten, angenähert werden. Diese sollen dadurch vermittelt werden, dass der Blick geschult wird, um zuvor nicht Gesehenes selbst zu erkennen.

Zudem geht das Konzept des Verstehen Lehrens über die Entwicklungsaufgaben (Gruschka 1985) hinaus, weil es nicht nur um die subjektive Bildungsbiographie geht, sondern auch bearbeitet werden kann, wie extern vorgegebene Inhalte (des Curriculums) vermittelt werden können. Ebenso könnten pädagogische Antinomien reflektiert werden. Dies ist sowohl für die Lehrkräfte an beruflichen Fachschulen relevant, als auch für die späteren Erzieher*innen.

\subsection{Sozialpädagogische Dimensionen guten Unterrichts}

Eine weitere Entwicklungslinie kann aufbauend auf die Analysen in A. Gruschkas (1985) Erzieher*innen Studie vorgenommen werden. Wie dargestellt, hat A. Gruschka (1985) die Bewältigung der beruflichen Entwicklungsaufgaben in unterschiedlichen Niveaustufen dargestellt. Im abschließenden Teil der Arbeit versucht A. Gruschka die Bewältigung der Entwicklungsaufgaben mit zentralen Unterrichtsvariablen zu erklären und nutzt hierfür Regressionsanalysen. Seine Ergebnisse zeigen, dass die Begründungen der Schüler*innen elaborierter sind, wenn ,diese in ihrem Unterricht verbindlich angeleitet, dabei aber auch tolerant ermutigt worden sind, ihre eigenen praktischen Motive umzusetzen“ (Gruschka 1985, S. 462), 
deren Unterricht an die Entwicklungsaufgaben anknüpft, und deren Lehrkräfte ,,simulativ die Konflikte [...] thematisieren“ (Gruschla 1985, S. 462), auf die sie im späteren Beruf stoßen können. Auch die Gruppenkonstellation scheint wichtig. Sind die Orientierungen der Schüler*innen zu einheitlich, kommt es seltener zu Auseinandersetzungen über die Entwicklungsaufgaben, als in heterogenen Gruppen.

Auch wenn A. Gruschka (1985) diese Ergebnisse etwas relativiert - und möglicherweise mit dem quantitativen Teil der Arbeit heute nicht mehr konform geht scheint die hier vorgestellte Studie angesichts des Mangels an Forschung zu didaktischen Situationen ein Ausgangspunkt für eine Weiterentwicklung einer Didaktik der Sozialpädagogik bieten zu können.

Das Konzept der Basisdimensionen guten Unterrichts von E. Klieme (2019) und Kolleg*innen könnte hier ein weiterer Ausgangspunkt sein, trotz (in Teilen berechtigter) Kritik an den quantifizierenden Lehr-Lern-Forschungsansätzen (oder der evidenzbasierten Forschung im Allgemeinen) (u. a. Tröhler 2015; Forster 2014; Meyer 2013; Gruschka 2012, 2006; Münch 2009).

Die drei Basisdimensionen guten Unterrichts sollten jedoch nicht einfach nur übernommen werden, sondern müssen fachbezogen weiterentwickelt und adaptiert werden (Klieme und Rakoczy 2008). Auch eine Erweiterung um sozialpädagogische Qualitätsmerkmale ist denkbar. Für den Kontext von Ganztagsschulen liegen beispielsweise Arbeiten vor, die neben Classroom Management, Motivationsunterstützung und kognitiver Aktivierung auch Partizipationsmöglichkeiten, Alltagsorientierung sowie drei Formen von Anerkennung - individuelle, solidarische, rechtliche - betrachten und zeigen können, dass diese in nicht-unterrichtlichen Angeboten und im Unterricht bedeutsam sind (Vieluf und Sauerwein 2018; Sauerwein 2018, 2017). Damit könnte eingeholt werden, dass Didaktik der Sozialpädagogik sich nicht nur in der Vermittlung, sondern auch in der Praxis stärker an den lebensweltlichen Hintergründen ihrer Adressat*innen orientieren muss, als die Schulpädagogik (Steinbacher 2018). Dies wäre auch im Unterricht an Fachschulen zu berücksichtigen und entsprechend in der Ausbildung von Lehrkräften.

A. Gruschkas (1985) Idee, Kompetenzen nicht über standardisierte Tests, sondern als Stufen der Elaboration unterschiedlicher Entwicklungsaufgaben zu rekonstruieren, scheint hier eine Möglichkeit darzustellen, eine genuin ,sozialpädagogische Antwort auf die Komplexität des späteren Berufsfeldes zu finden. Letztlich versteht A. Gruschka (1985) in seiner Studie Kompetenzen zwar ähnlich wie in den Bildungsstandards, als verfügbare Fertigkeiten und Fähigkeiten Probleme zu lösen und in verschiedenen Situationen erfolgreich nutzen zu können (Klieme et al. 2003). Die rekonstruierten Elaborationen lassen jedoch auch ein strukturtheoretisches Professionsverständnis $\mathrm{zu}$, da sich in den Antworten auch die Reflexion der Antinomien pädagogischen Handelns widerspiegelt. Natürlich wird hiermit nicht postuliert, dass die pädagogischen Antinomien (im Studium oder Unterricht) aufgelöst, jedoch im Sinne einer Qualifizierung und Professionalisierung auf unterschiedlichen Niveaustufen bearbeitet werden können. Denn gerade in dem Umgang mit diesen Antinomien bildet sich Professionalität heraus. Diese Idee könnte letztlich eine Integration der unterschiedlichen professionstheoretischen Verständnisse ermöglichen und zugleich die Herausforderungen oftmals nicht planbarer sozialpädagogischer Situationen berücksichtigen. 
Colin Cramer et al. (2019) haben dies als Meta-Reflexivität bezeichnet, die nicht auf die Generierung von Professionswissen und Kompetenz ausgerichtet ist. „Eine meta-reflexive Lehrperson zeichnet sich dadurch aus, dass sie in der (universitären) Lehrerbildung möglichst umfassende, exemplarisch-typisierende Situationsdeutungen gewonnen hat und im Handlungsfeld Schule möglichst angemessen situative Deutungen - falls eine näherungsweise Passung gegeben ist, dann unter Rekurs auf die exemplarisch-typisierenden Situationsdeutungen - vornimmt und nach diesen handelt" (Cramer et al. 2019, S. 412).

\section{Resümee}

Die hier dargestellten Konturen für eine Weiterentwicklung einer Didaktik der Sozialpädagogik können geeignet sein, den eingangs dargestellten Herausforderungen zu begegnen und Konsequenzen für die Ausbildung von Lehrer*innen für Fachschulen der Sozialpädagogik zu ziehen. Didaktik ist hierbei als ,in wechselseitiger Beziehung stehendes Lehren und Lernen“ (Karber 2017, S. 86) zu verstehen.

Aufgrund der dargestellten Scholarisierungsprozesse ist eine Kompetenzorientierung der Erzieher*innen Ausbildung und letztlich damit auch der Ausbildung der Lehrkräfte an Fachschulen für Sozialpädagogik nicht mehr zu ignorieren. Freilich kann Kompetenzorientierung sozialtechnologisch missverstanden werden. In diesem Kontext kann es nicht zielführend sein, diese und die mit ihr verknüpfte Lehr-LernForschung zurückzuweisen (Karsten und Kubandt 2017), sondern Kompetenzorientierung sozialpädagogisch zu wenden. Kompetenzen können sich in diesem Sinne auch in der Entwicklung einer professionellen Identität widerspiegeln, wenn sich etwa Lehrkräfte wie auch zukünftige Erzieher*innen den Antinomien ihrer Berufspraxis bewusst werden. Dies wäre aber nicht über standardisierte Test zu erheben, sondern über reflexive Argumentationsmuster, womit auch die von N. Göddertz und A. Karber (2019) angemahnte Analyse und Kritikfähigkeit berücksichtigt werden kann.

Zugleich könnte hierüber Deprofessionalisierungstendenzen (unterstützt durch empirische Forschung) entgegengetreten werden. Einerseits auf Seiten der Lehrkräfte, wenn gezeigt werden kann, dass ein Studium der Sozialdidaktik tatsächlich die Entwicklung einer professionellen Identität von zukünftigen Erzieher*innen anregt. Andererseits, wenn für die Ausbildung der der zukünftigen Erzieher*innen Hinweise erarbeitet werden, welche Grundlagen es bedarf, um Reflexivität zu vermitteln. Damit könnte der mangelnden Bedeutung einer Didaktik der Sozialpädagogik entgegengetreten werden, wobei diese Neujustierung von empirischen Studien begleitet werden muss und basierend auf diesen Erkenntnissen weiter überarbeitet werden sollte.

Funding Open Access funding enabled and organized by Projekt DEAL.

Open Access Dieser Artikel wird unter der Creative Commons Namensnennung 4.0 International Lizenz veröffentlicht, welche die Nutzung, Vervielfältigung, Bearbeitung, Verbreitung und Wiedergabe in jeglichem Medium und Format erlaubt, sofern Sie den/die ursprünglichen Autor(en) und die Quelle ord- 
nungsgemäß nennen, einen Link zur Creative Commons Lizenz beifügen und angeben, ob Änderungen vorgenommen wurden.

Die in diesem Artikel enthaltenen Bilder und sonstiges Drittmaterial unterliegen ebenfalls der genannten Creative Commons Lizenz, sofern sich aus der Abbildungslegende nichts anderes ergibt. Sofern das betreffende Material nicht unter der genannten Creative Commons Lizenz steht und die betreffende Handlung nicht nach gesetzlichen Vorschriften erlaubt ist, ist für die oben aufgeführten Weiterverwendungen des Materials die Einwilligung des jeweiligen Rechteinhabers einzuholen.

Weitere Details zur Lizenz entnehmen Sie bitte der Lizenzinformation auf http://creativecommons.org/ licenses/by/4.0/deed.de.

\section{Literatur}

Baumert, J., \& Kunter, M. (2006). Stichwort: Professionelle Kompetenz von Lehrkräften. Zeitschrift für Erziehungswissenschaften, 9(4), 469-520.

Beck, U., \& Bonß, W. (Hrsg.). (1989). Weder Sozialtechnologie noch Aufklärung? Analysen zur Verwendung sozialwissenschaftlichen Wissens. Frankfurt a. Main: Suhrkamp.

Beher, K., Hoffmann, H., \& Rauschenbach, T. (1999). Das Berufsbild der ErzieherInnen. Vom fächerorientierten zum tätigkeitsorientierten Ausbildungskonzept. Neuwied: Luchterhand.

Breidenstein, G. (2010). Überlegungen zu einer Theorie des Unterrichts. Zeitschrift für Pädagogik, 56(6), 869-887.

Cloos, P., \& Hoffmann, H. (2001). Die Ausbildung der AusbildnerInnen. Zum Studium des Lehramtes an Fachschulen/Fachakademien für Sozialpädagogik. In H. Hoffmann (Hrsg.), Studien zur Qualitätsentwicklung von Kindertagesstätten (S. 51-96). Neuwied, Luchterhand: Beltz.

Cramer, C., Harant, M., Merk, S., Drahmann, M., \& Emmerich, M. (2019). Meta-Reflexivität und Professionalität im Lehrerinnen- und Lehrerberuf. Zeitschrift für Pädagogik, 65(3), 401-423.

Creemers, B.P.M., \& Kyriakidēs, L. (2008). The dynamics of educational effectiveness. A contribution to policy, practice and theory in contemporary schools (Contexts of learning). London, New York: Routledge.

Drexl, D., Born-Rauchenecker, E., \& Kalicki, B. (2019). Naturwissenschaftliche Einstellungen angehender pädagogischer Fachkräfte. Frühe Bildung, 8(1), 30-36.

Forster, E. (2014). Kritik der Evidenz. Das Beispiel evidence-informed policy research der OECD. Zeitschrift für Pädagogik, 60(6), 890-907.

Fröhlich-Gildhoff, K., Nentwig-Gesemann, I., \& Pietsch, S. (2011). Kompetenzorientierung in der Qualifizierung frühpädagogischer Fachkräfte. Eine Expertise der Weiterbildungsinitiative Frühpädagogische Fachkräfte (WiFF) (Ausbildung). Bd. 19. München: DJI e. V..

Frommberger, D. \& Lange, S. (2018). Zur Ausbildung von Lehrkräften für berufsbildende Schulen. WISO DISKURS Nr. 04. Bonn: Friedrich Ebert Stiftung (Hrsg.).

Gängler, H., \& Liebig, M. (2017). Lehren und Lernen in der Sozialpädagogik. In F. Kessl, E. Kruse, S. Stövesand \& W. Thole (Hrsg.), Soziale Arbeit - Kernthemen und Problemfelder (S. 253-259). Opladen: Barbara Budrich.

Gängler, H., \& Wustmann, C. (2004). Die Sozialpädagogik und ihre Didaktik. In B. Fegebank \& H. Schanz (Hrsg.), Arbeit - Beruf - Bildung in Berufsfeldern mit personenorientierten Dienstleistungen (S. 95-115). Baltmannsweiler: Schneider-Verl. Hohengehren.

Göddertz, N., \& Karber, A. (2019). Berufliche Bildung Sozialpädagogik - Eine Spurensuche didaktischer Prinzipien. Soziale Passagen, 11(1), 65-80.

Gruschka, A. (1985). Wie Schüler Erzieher werden. Studie zur Kompetenzentwicklung und fachlichen Identitätsbildung in einem doppeltqualifizierenden Bildungsgang des Kollegschulversuchs NW. Wetzlar: Büchse der Pandora.

Gruschka, A. (2006). Bildungsstandards oder das Versprechen, Bildungstheorie in empirischer Bildungsforschung aufzuheben. In U. Frost (Hrsg.), Unternehmen Bildung. Die Frankfurter Einsprüche und kontroverse Positionen zur aktuellen Bildungsreform. Vierteljahrsschrift für wissenschaftliche Pädagogik Sonderheft. Paderborn: Schöningh.

Gruschka, A. (2011). Verstehen lehren. Ein Plädoyer für guten Unterricht. Ditzingen: Reclam.

Gruschka, A. (2012). Missratener Fortschritt - Glänzende Geschäfte. Vierteljahresschrift für wissenschaftliche Pädagogik, 1, 96-109. 
Gruschka, A. (2013). Unterrichten - eine pädagogische Theorie auf empirischer Basis. Leverkusen: Barbara Budrich.

Gruschka, A. (2017). Was heißt „,bildender Unterricht“? Zeitschrift für interpretative Schul- und Unterrichtsforschung, 5(1), 77-92.

Gruschka, A., Hesse-Lenz, C., Michely-Weirich, H., \& Schomacher, H. (1995). Aus der Praxis lernen. Berlin: Cornelsen.

Hamre, B. K., Pianta, R.C., Mashburn, A. J., \& Downer, J. T. (2007). Building a science of classrooms: Application of the CLASS framework in over 4,000 U.S. early childhood and elementary classrooms. University of Virginia: Foundation for Child Development.

Hasselhorn, M., \& Gold, A. (2012). Pädagogische Psychologie. Erfolgreiches Lernen und Lehren (3. Aufl.). Stuttgart: Kohlhammer.

Helsper, W. (2007). Eine Antwort auf Jürgen Baumerts und Mareike Kunters Kritik am strukturtheoretischen Professionsansatz. Zeitschrift für Erziehungswissenschaft, 10(4), 567-579.

Helsper, W. (2014). Lehrerprofessionalität - der strukturtheoretische Professionsansatz zum Lehrerberuf. In E. Terhart, H. Bennewitz \& M. Rothland (Hrsg.), Handbuch der Forschung zum Lehrerberuf (2. Aufl. S. 216-240). Münster: Waxmann.

Helsper, W. (2016). Antinomien und Paradoxien im professionellen Handeln. In M. Dick, W. Marotzki \& H. A. Mieg (Hrsg.), Handbuch Professionsentwicklung (S. 50-62). Bad Heilbrunn: Verlag Julius Klinkhardt.

Helsper, W., \& Tippelt, R. (2011). Ende der Profession und Professionalisierung ohne Ende? Zwischenbilanz einer unabgeschlossenen Diskussion. Zeitschrift für Pädagogik, Beiheft, (57), 268-288.

Hörster, R. (2018). Kasuistik. In H.-U. Otto, H. Thiersch, R. Treptow \& H. Ziegler (Hrsg.), Handbuch Soziale Arbeit. Grundlagen der Sozialarbeit und Sozialpädagogik (6. Aufl. S. 1563-1571). München: Ernst Reinhardt Verlag.

Idel, T.-S., Reh, S., \& Rabenstein, K. (2014). Pädagogische Ordnungen als Fall. Fallarbeit und Professionalisierung aus praxistheoretischer Sicht. In I. Pieper, P. Frei, K. Hauenschild \& B. Schmidt-Thieme (Hrsg.), Was der Fall ist. Beiträge zur Fallarbeit in Bildungsforschung, Lehramtsstudium, Beruf und Ausbildung (S. 75-88). Wiesbaden: Springer.

Karber, A. (2014). Sozialpädagogik und Didaktik. Herausforderung aus einem ungeklärten Verhältnis. Grundlagen der sozialen Arbeit, Bd. 33. Baltmannsweiler: Schneider-Verl. Hohengehren.

Karber, A. (2017). Sozialdidaktik als Berufsfelddidaktik - Didaktik in sozialpädagogischen Bildungsgängen aus der Perspektive der beruflichen Fachrichtung Sozialpädagogik. In M.-E. Karsten \& M. Kubandt (Hrsg.), Lehramtsstudium Sozialpädagogik. Eine Bestandsaufnahme nach 20 Jahren (S. 85-96). Opladen: Verlag Barbara Budrich.

Karber, A., \& Wustmann, C. (2015). Forschendes Lehren und Lernen. Perspektiven der Akteurinnen und Akteure in den Blick nehmen und erforschen. In R. Egger, C. Wustmann \& A. Karber (Hrsg.), Forschungsgeleitete Lehre in einem Massenstudium. Bedingungen und Möglichkeiten in den Erziehungsund Bildungswissenschaften (S. 37-54). Wiesbaden: Springer VS.

Karsten, M.-E. (2017). Bildungswege zur „ganzen“ Berufs- und Professionsvielfalt im Arbeitsmarkt der Sozialen Arbeit und Sozialpädagogik. In M.-E. Karsten \& M. Kubandt (Hrsg.), Lehramtsstudium Sozialpädagogik. Eine Bestandsaufnahme nach 20 Jahren (S. 21-31). Opladen: Verlag Barbara Budrich.

Karsten, M.-E., \& Kubandt, M. (2017). Lehramtsstudium Sozialpädagogik - Potenziale zur Professionalisierung des gesamten Feldes der Sozialen Arbeit - eine Einführung. In M.-E. Karsten \& M. Kubandt (Hrsg.), Lehramtsstudium Sozialpädagogik. Eine Bestandsaufnahme nach 20 Jahren (S. 11-20). Opladen: Verlag Barbara Budrich.

Klieme, E. (2019). Unterrichtsqualität. In M. Gläser-Zikuda, M. Harring \& C. Rohlfs (Hrsg.), Handbuch Schulpädagogik 1. Aufl. Stuttgart: UTB.

Klieme, E., \& Rakoczy, K. (2008). Empirische Unterrichtsforschung und Fachdidaktik. Outcome-orientierte Messung und Prozessqualität des Unterrichts. Zeitschrift für Pädagogik, 54(2), 222-237.

Klieme, E., Avenarius, H., Blum, W., Döbricht, P., Gruber, H., \& Prenzel, M. (2003). Zur Entwicklung nationaler Bildungsstandards. Bonn: Bundesministerium für Bildung und Forschung (BMBF).

Klieme, E., Schümer, G., \& Knoll, S. (2001). Mathematikunterricht in der Sekundarstufe I: Aufgabenkultur und Unterrichtsgestaltung. In E. Klieme \& J. Baumert (Hrsg.), TIMMS - Impulse für Schule und Unterricht (S. 43-57). Bonn: BMBF.

König, A. (2016). Die Dominanz der Fachschulen für Sozialpädagogik im Zuge der (Teil-)Akademisierung im Arbeitsfeld Kindertageseinrichtung - Analysen zur Eigenlogik des Feldes. In: bwp@Berufs-und Wirtschaftspädagogik - online, Ausgabe 31, 1-17. Online: http://www.bwpat.de/ausgabe31/koenig_ bwpat31.pdf. 
König, A., Kratz, J., Stadler, K. \& Uihlein, C. (2018). Aktuelle Entwicklungen in der Ausbildung von Erzieherinnen und Erziehern an Fachschulen für Sozialpädagogik. Organisationsformen, Zulassungsvoraussetzungen und Curricula - eine Dokumentenanalyse: Eine Studie der Weiterbildungsinitiative Frühpädagogische Fachkräfte (WiFF) (Ausbildung, Band 29). München: DJI e. V.

Kruse, E. (2017). Aus- und Weiterbildung in der Sozialen Arbeit. In F. Kessl, E. Kruse, S. Stövesand \& W. Thole (Hrsg.), Soziale Arbeit - Kernthemen und Problemfelder (S. 182-194). Opladen: Verlag Barbara Budrich.

Küls, H. (2010). Lernfelder in der Sozialpädagogik - Überlegungen zur Zukunft eines Curriculums. In A. Fischer \& G. Hahn (Hrsg.), Schule - der Zukunft voraus. Was wäre, wenn...? Lese- und Lehrbuch für zukunftsorientierte Ansätze in der beruflichen Bildung (S. 31-44). Baltmannsweiler: SchneiderVerl. Hohengehren.

Kultusminister Konferenz (KMK) (2017). Kompetenzorientiertes Qualifikationsprofil für die Ausbildung von Erzieherinnen und Erziehern an Fachschulen und Fachakademien. Beschluss der Kultusministerkonferenz vom 01.12.2011 i.d.F. vom 24.11.2017

Kunter, M., \& Trautwein, U. (Hrsg.). (2013). Psychologie des Unterrichts. Paderborn: Schöningh.

Kyriakides, L., Christoforou, C., \& Charalambous, C. Y. (2013). What matters for student learning outcomes: a meta-analysis of studies exploring factors of effective teaching. Teaching and Teacher Education, 36, 143-152.

Liebig, M. (2016). Die Fachschulausbildung zur Erzieherin/zum Erzieher unter Druck: Entwicklungslinien in der Ausbildung frühpädagogischen Fachpersonals. In: bwp@ Berufs- und Wirtschaftspädagogik online, Ausgabe 31, 1-20. Online: http://www.bwpat.de/ausgabe31/liebig_bwpat31.pdf.

Liebig, M., \& Gängler, H. (2018). Lehrerinnen- und Lehrerbildung als Persönlichkeitsbildung. bwp@Berufs- und Wirtschaftspädagogik - online, Ausgabe 34, 1-14. Online: http://www.bwpat.de/ausgabe34/ liebig_gaengler_bwpat34.pdf. Zugegriffen: 10. Dez. 2019.

Messmer, R. (2014). Denken Lehrpersonen anders als Didaktiker/innen schreiben? In I. Pieper, P. Frei, K. Hauenschild \& B. Schmidt-Thieme (Hrsg.), Was der Fall ist. Beiträge zur Fallarbeit in Bildungsforschung, Lehramtsstudium, Beruf und Ausbildung (S. 59-74). Wiesbaden: Springer.

Meyer, H.-D. (2013). PISA, power, and policy: the emergence of global educational governance; [conference at the University at Albany (the State University of New York) in December 2011. Oxford studies in comparative education, Bd. 23,1. Oxford: Symposium Books.

Münch, R. (2009). Globale Eliten, lokale Autoritäten. Bildung und Wissenschaft unter dem Regime von PISA, McKinsey \& Co. Frankfurt a. Main: Suhrkamp.

Natorp, P. (1899). Sozialpädagogik. Theorie der Willenserziehung auf der Grundlage der Gemeinschaft. Stuttgart: Fr. Frommanns Verlag.

Oevermann, U. (1996). Theoretische Skizze einer revidierten Theorie professionellen Handelns. In A. Combe \& W. Helsper (Hrsg.), Pädagogische Professionalität. Untersuchungen zum Typus pädagogischen Handelns (S. 70-182). Frankfurt a. Main: Suhrkamp.

Pianta, R.C., \& Hamre, B. K. (2009a). Conceptualization, measurement, and improvement of classroom processes: standardized observation can leverage capacity. Educational Researcher, 38(2), 109-119.

Pianta, R. C., \& Hamre, B. K. (2009b). Classroom processes and positive youth development: Conceptualizing, measuring, and improving the capacity of interactions between teachers and students. New Directions for Youth Development, 2009(121), 33-46.

Pianta, R.C., La Paro, K.M., \& Hamre, B.K. (2008). Classroom assessment scoring system (CLASS) manual, $K-3$. Baltimore: Paul H. Brookes Publishing Co.

Pietsch, M. (2010). Evaluation von Unterrichtsstandards. Zeitschrift für Erziehungswissenschaft, 13(1), 121-148.

Pohle, L., Hosoya, G., Loftfield, C., \& Jenßen, L. (2019). Indicators measuring preschool teachers' stimulation quality. Zeitschrift für Pädagogik, 65(4), 525-541.

Praetorius, A.-K., Klieme, E., Herbert, B., \& Pinger, P. (2018). Generic dimensions of teaching quality. The German framework of three basic dimensions. ZDM, 50(3), 407-426.

Prange, K. (2011). Didaktik und Methodik. In J. Kade, W. Helsper, M. Lüders, B. Egloff, F.-O. Radtke \& W. Thole (Hrsg.), Pädagogisches Wissen. Erziehungswissenschaft in Grundbegriffen (S. 183-190). Stuttgart: Kohlhammer.

Reusser, K. (2008). Empirisch fundierte Didaktik - didaktisch fundierte Unterrichtsforschung. Eine Perspektive zur Neuorientierung der Allgemeinen Didaktik. In M. A. Meyer, S. Hellekamps \& M. Prenzel (Hrsg.), Perspektiven der Didaktik. Zeitschrift für Erziehungswissenschaft. Sonderheft, (Bd. 9, S. 219-237). Wiesbaden: VS. 
Rudolph, B. (Hrsg.). (2012). Das Berufsbild der Erzieherinnen und Erzieher im Wandel - Zukunftsperspektiven zur Ausbildung aus Sicht der Fachschulleitungen. Eine Studie der Weiterbildungsinitiative Frühpädagogische Fachkräfte (WiFF). WiFF-Studien Ausbildung, Bd. 14. München: DJI e. V..

Sandilos, L.E., DiPerna, J.C., \& Investi, T. F. (2014). Measuring quality in kindergarten classrooms. Structural analysis of the classroom assessment scoring system (CLASS K-3). Early Education and Development, 25(6), 894-914.

Sauerwein, M.N. (2017). Qualität in Bildungssettings der Ganztagsschule. Über Unterrichtsforschung und Sozialpädagogik. Weinheim: Beltz Juventa.

Sauerwein, M.N. (2018). Partizipation in der Ganztagsschule - vertiefende Analysen. Zeitschrift für Erziehungswissenschaft, 62(6), 830-855.

Sauerwein, M.N., \& Klieme, E. (2016). Anmerkungen zum Qualitätsbegriff in der Bildungsforschung. Schweizerische Zeitschrift für Bildungswissenschaften, 38(3), 459-478.

Steinbacher, E. (2018). Didaktik. In H.-U. Otto, H. Thiersch, R. Treptow \& H. Ziegler (Hrsg.), Handbuch Soziale Arbeit. Grundlagen der Sozialarbeit und. Sozialpädagogik, (Bd. 6, S. 256-262). München: Ernst Reinhardt Verlag.

Terhart, E. (2018). Allgemeine Didaktik und Unterrichtstheorie im deutschsprachigen Raum: Beobachtungen und Einordnungen. In D. Benner, H. Meyer, Z. Peng \& Z. Li (Hrsg.), Beiträge zum chinesischdeutschen Didaktik-Dialog. Bad Heilbrunn: Verlag Julius Klinkhardt.

Tröhler, D. (2015). The medicalization of current educational research and its effects on education policy and school reforms. Discourse: Studies in the Cultural Politics of Education, 36(5), 749-764.

Vieluf, S., \& Sauerwein, M.N. (2018). Does a lack of teachers' recognition of students with migration background contribute to achievement gaps? European Educational Research Journal, 7, 1-25.

Vriesen, J. (2019). Studienskizze und Studienprojekt. Umsetzung des Forschenden Lernens im Rahmen des Praxissemesters in den Bildungswissenschaften an der Technischen Universität Dortmund. In M. Degeling, N. Franken \& S. Freund (Hrsg.), Herausforderung Kohärenz: Praxisphasen in der universitären Lehrerbildung. Bildungswissenschaftliche und fachdidaktische Perspektiven (S. 149-159). Bad Heilbrunn: Verlag Julius Klinkhardt.

Wernet, A. (2014). Überall und nirgends. Ein Vorschlag zur professionalisierungstheoretischen Verortung des Lehrerberufs. In C. Leser, T. Pflugmacher, M. Pollmanns, J. Rosch \& J. Twardella (Hrsg.), Zuneignung. Pädagogik und Widerspruch (S. 77-96). Leverkusen-Opladen: Barbara Budrich.

Weyland, U. (2019). Forschendes Lernen in Langzeitpraktika. Hintergründe, Chancen und Herausforderungen. In M. Degeling, N. Franken \& S. Freund (Hrsg.), Herausforderung Kohärenz: Praxisphasen in der universitären Lehrerbildung. Bildungswissenschaftliche und fachdidaktische Perspektiven (S. 25-64). Bad Heilbrunn: Verlag Julius Klinkhardt.

Wildt, J. (2005). Auf dem Weg zu einer Didaktik der Lehrerbildung? Beiträge zur Lehrerinnen- und Lehrerbildung, 23(2), 183-190.

Zierer, K. (2012). Bildung und Kompetenz. Eine kritisch-konstruktive Analyse auf Grundlage einer eklektischen Didaktik. Vierteljahresschrift Für wissenschaftliche Pädagogik, 88(1), 30-53. https://doi.org/ 10.1163/25890581-088-01-90000005. 